\title{
Design and Control of a Quadruple-Input Double-Output Coherent Radar Test Platform Composed of Test \& Measurement Equipment and Custom Hardware
}

\author{
Yanmin Chen, Kuoye Han and Mian Jiang \\ Information Science Academy(ISA), China Electronics Technology Group Corporation(CETC), Beijing 100098, P. R. China
}

\begin{abstract}
A radar test platform composed of commercial off-the-shelf test and measurement equipment and custom hardware has been built. This platform, which enables production of dual-channel coherent radar transmitting signals with a bandwidth up to $500 \mathrm{MHz}$, and reception of four parallel channels of RF injection signals or echoes, can simulate various types of radar emitters or to simulate the multiple modes of operation for a single type radar. A software is also programmed with $\mathrm{C}++$ language to control the waveforms, frequencies, phases, time delays and all other aspects of the test platform's operation at all stages. At last, an application example is given when the platform is used for ISAR imaging verification.
\end{abstract}

\section{Introduction}

Nowadays, the performance of telecommunication systems and radar systems has been greatly improved, due to the application of various new technologies, one of which is the technology of emitting and receiving multichannel phase coherent signals. During the testing stage of the receivers of satellite communication systems, mobile communication systems, MIMO systems and phased array radar systems, it happened plenty of times that a set of multi-channel phase coherent signals is needed as a stimulus to the corresponding subsystems under test. These phase coherent signals are supposed to be with precisely adjustable frequencies, phases, and time delays, that can simulate the real-world signal characteristics and are used to test and evaluate the performance of the receivers or the subsystems. Therefore, it requires that a test platform capable of producing multi-channel phase coherent RF or IF signals flexibly and conveniently in the laboratory be built in order to implement the functions as mentioned above.

In this paper, we focus on the development and generation of actual radar test signals used to exercise all of the radar's functional stages either individually or the entire radar system collectively, for example, simulating the characteristics of a set of microwave signals at different input ports of array antennas, simulating the input signals of a digital beamforming network, or providing multi-channel coherent signal injections to the up- or down-conversion modules of a radar system and subsequently receiving and analysing the responses of the device under test. Signals can be applied at RF, IF or baseband to evaluate or characterize a radar or its subsystems.
A radar test platform composed of commercial offthe-shelf test and measurement equipment and custom hardware is designed and built. This platform supports the ability to generate dual-channel coherent transmitting signals and to receive four channels of test responses. By precisely controlling the waveforms, frequencies, phases, time delays and the signal flows, it can simulate various types of radar emitters or to simulate the multiple modes of operation for a single type of radar. The system design issues including the structure and functions, the composition of each functional module, the trigger and synchronization mechanism, the approach of maintaining phase consistency, and the control strategy and method are presented in the subsequent sections. At last, one of the I/O channels of the test platform is utilized, over which microwave pulses are sent and received, and an ISAR image of a maneuvering vehicle target is obtained, which collectively demonstrates the high quality of signals produced and good performance of the test platform.

\section{Radar test platform design}

\subsection{System structure and functions}

The radar test platform consists of the following modules: the dual-channel baseband signal generation module, the dual-channel quadrature (I/Q) modulation module, the frequency and timer module, the receiver downconversion module, the AD sampling \& recording module, the high power amplifier module and the antenna module, the power supply module and an industrial personal computer. Most core modules are made up of 


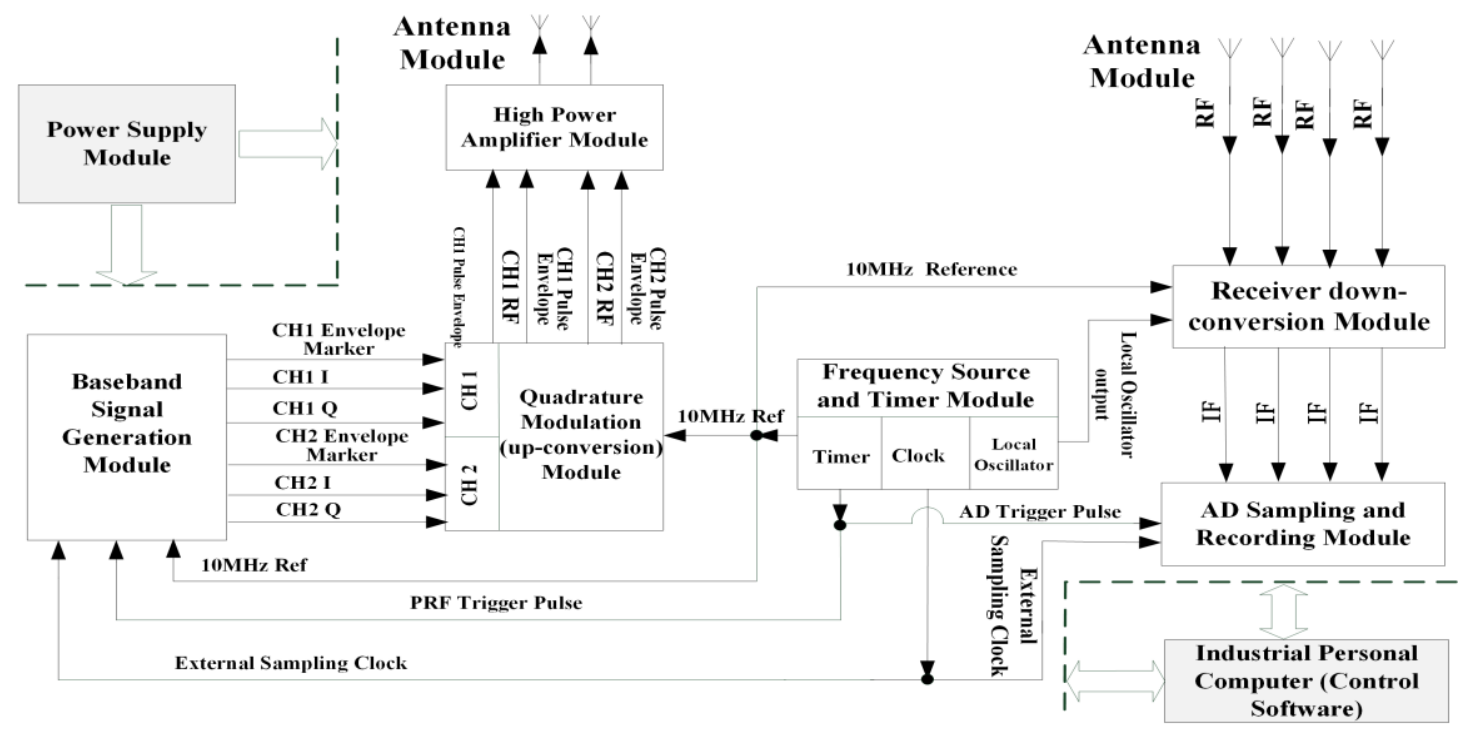

Figure 1. Block diagram of the radar test platform.

commercial off-the-shelf test \& measurement equipment, which shows the broad capability of readily available hardware from a laboratory. Figure 1 is a block diagram of hardware modules in a configuration to support the ability to generate dual-channel coherent transmitting signals and to receive four channels of responses.

\subsection{Baseband signal generation}

The baseband signal generation module is based on arbitrary waveform generators (AWGs), where multiple set of I/Q waveform sequences can be stored in advance and then played as analogue signals with the actions of external triggers and clock. Flexible parameter setting gives the user choices of various types of waveforms including the monochromatic wave, linear frequency modulation (LFM) signal, and digital modulation signals frequently used in telecommunication such as BPSK, QPSK and etc.

As shown in Figure 2, two Agilent N6030A AWGs are configured to form a dual-channel baseband signal generation module. One N6030A unit is designated as the

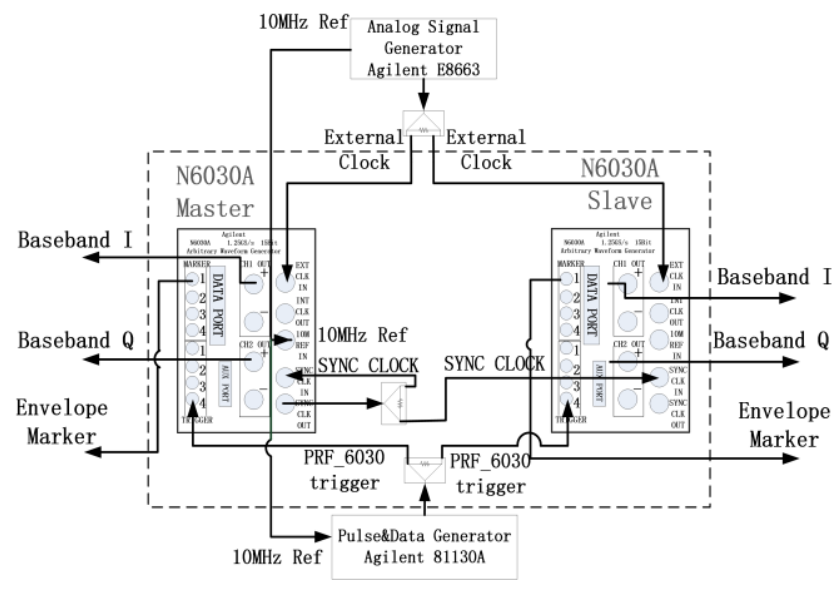

Figure 2. Configurations of the dual-channel baseband signal generation module.

Master and the other one is designated as the Slave. These two AWGs are synchronized by sharing a common external clock and a common trigger, where either the trigger or the clock is split with low skew and distributed with cables of equal length to both units. The I or Q channel of each N6030A can produce signals range from $\mathrm{DC}$ to $500 \mathrm{MHz}$, and the two quadrature signal components are synthesized to a signal with more broad bandwidth at the quadrature modulation module.

\subsection{Quadrature (I/Q) modulation: up-conversion}

The quadrature (I/Q) modulation module is mainly made up of two Agilent E8267D PSG signal generators, one designated as Master and the other as Slave, corresponding to the two baseband signal generation channels, as shown in Figure 3. 
The Master E8267D outputs a carrier frequency signal through an HCC option module installed on it, and this signal is split into two equivalents by a U3035P distribution network, then fed back to the input port of

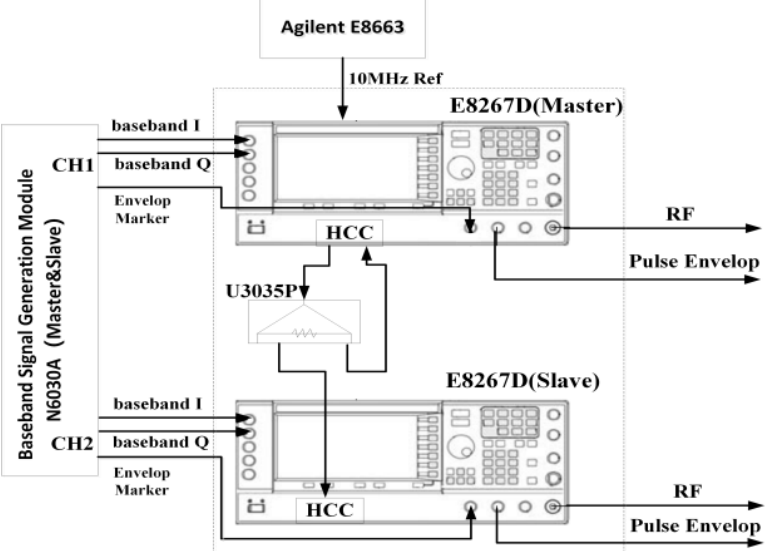

Figure 3. Configurations of the dual-channel baseband quadrature modulation module.

each E8267D's HCC option. By this means, the carrier frequency signals to the Master and the Slave channels maintain the same frequency and phase. It is also worth noting that an 015/016 wideband option is installed on the equipment to enable a production of signal bandwidth up to $1 \mathrm{GHz}$.

\subsection{Receiver down-conversion}

The receiver of the platform is designed to have four parallel channels, therefore, an Agilent N5280A fourchannel frequency converter as shown in Figure 4 is chosen, with custom low noise RF amplifiers, band-pass filters and IF amplifiers integrated with it to down convert a set of received (or injected) RF signals.

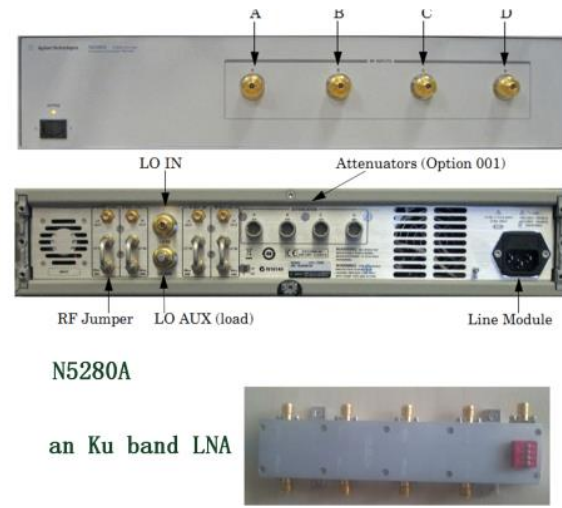

Figure 4. The N5280A four-channel frequency converter and a $\mathrm{Ku}$ band low noise amplifier.

\subsection{Other modules}

The frequency source and timer module is responsible for the generation of the triggers, the reference signals, the external clocks and the local oscillator. Here, an Agilent $81130 \mathrm{~A}$ pulse generator is used to produce the triggers to the baseband signal generation module and to the AD sampling and recording module; an Agilent E8663 analog signal generator is used to produce the external clock signal, and the local oscillator to the receiver downconversion module is produced by a R\&S SMR20 analog signal generator. It is worth noting that the quadrature (I/Q) modulation module has its own carrier frequency source (E8268Ds), rather than using the output of the frequency source and timer module.

The high power amplifier module in this test platform consists of several Agilent 83020 high power amplifiers, which have a maximum gain of $30 \mathrm{~dB}$ and can output signals with a maximum power of $30 \mathrm{dBm}$.

The AD sampling and recording module is a fourchannel custom module with a maximum sampling frequency of $2 \mathrm{GHz}$ and maximum transmission bandwidth of $500 \mathrm{MBps}$. It is designed to sample four IF down-converted IF signals simultaneously, then the sampled sequences are transmitted to the local industrial personal computer and digitally demodulated to $\mathrm{I} / \mathrm{Q}$ channels.

The users can choose different antennas according to the specific applications, for example,

\subsection{The synchronization mechanism and phase consistency}

All the modules in the test platform share a common 10 $\mathrm{MHz}$ reference signal to guarantee the phase consistency among different local oscillators. Then each module puts this reference signal through its phase-lock-loop frequency multiplier or division circuit to generate its own signals. We have mentioned that the phase consistency among different parallel channels are held by using a Master-Slave form, for example, in the quadrature modulation module, the master and slave E8267Ds share a common carrier frequency originated from the master.

\section{Access and control of the devices}

Most of the commercial off-the-shelf test and measurement equipment use drives of the IVI-C or IVICOM form and provide generalized Virtual Instrument Software Architecture (VISA) programming interface. The VISA is a Test \& Measurement industry standard communication API (Application Programming Interface) for use with test and measurement devices. Figure 5 is a flow chart to initiate VISA and communicate with an equipment. 


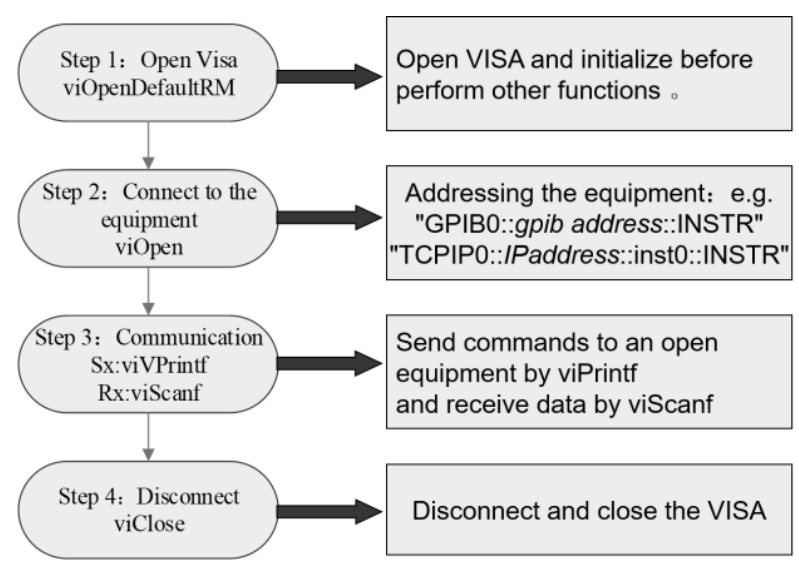

Figure 5. Control flow chart using VISA.

We developed a software, by which all the device of the test platform can be controlled individually and collectively. The software can adjust the signal parameters adaptively during the work period according to predefined patterns. It helps to simulate various types of radar emitters or to simulate the multiple modes of operation for a single type radar. The control software is programmed with $\mathrm{C} / \mathrm{C}++$ language and installed on an industrial personal computer. In addition, some signal/data processing functions are also integrated, e.g. digital quadrature demodulation, clutter filtering and pulse compression.

\section{ISAR imaging verification experiment}

An ISAR imaging verification experiment was carried out, where only one transceiver channel of the test platform is utilized. In Figure 6, the trigger pulse (yellow), the baseband I (green), baseband Q (blue), and the pulse envelop marker (pink) are displayed on an oscilloscope screen. Here, LFM I/Q baseband signal is generated from the AWG and transferred to the Quadrature (I/Q) modulation module for up-conversion on the carrier frequency. This transmission channel is pre-distortion calibrated to eliminate the I/Q imbalance and other channel imperfection. The platform works at $\mathrm{Ku}$ band and the measurement parameters are listed in Table 1.

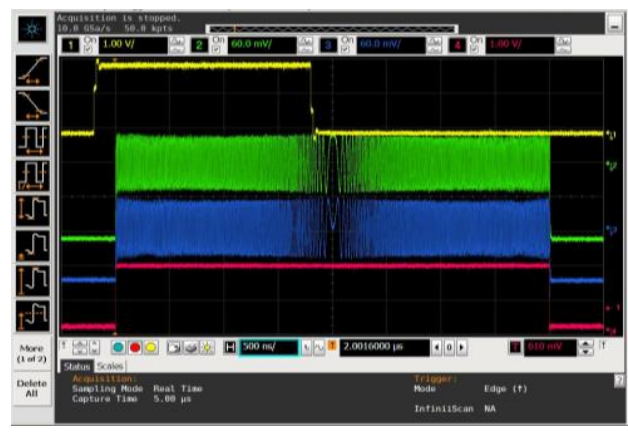

Figure 6. The trigger signal and signals generated by the baseband generation module.

Table 1. Measurement parameters.

\begin{tabular}{llll}
\hline \multicolumn{1}{c}{ Parameter } & value & Parameter & value \\
\hline Carrier frequency & $17 \mathrm{GHz}$ & $\begin{array}{l}\text { Frequency } \\
\text { bandwidth }\end{array}$ & $400 \mathrm{MHz}$ \\
PRF & $6000 \mathrm{~Hz}$ & Peak power & $0 \mathrm{dBm}$ \\
$\begin{array}{l}\text { LO frequency of } \\
\text { down conversion }\end{array}$ & $\begin{array}{l}16.8 \\
\mathrm{GHz}\end{array}$ & $\begin{array}{l}\text { AD sampling } \\
\text { frequency }\end{array}$ & $960 \mathrm{MHz}$ \\
\hline
\end{tabular}

We use two horn antennas, one for transmitting and the other for receiving, rather than using only one antenna and a circulator for transceiver channel switching. Before acquiring the ISAR echo data of a moving vehicle, we first turn the antenna beam at a corner reflector, see Figure 7, the range profile of this featured strong target is presented and the range resolution measured is close to the theoretical value of $0.375 \mathrm{~m}$. The response of this corner reflector can be saved as a reference for latter matched filtering processing. Also note that the first strong compressed pulse originates from direct coupling from the transmitting antenna to the receiving antenna, which should be supressed in practice.

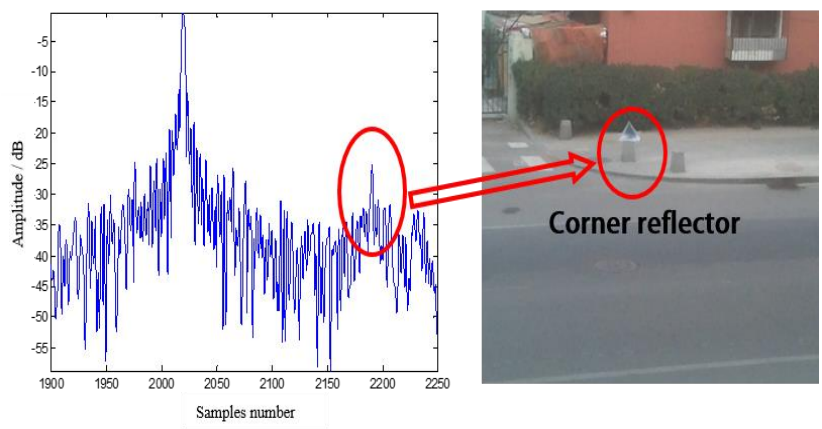

Figure 7. Range profile of a triangular corner reflector.

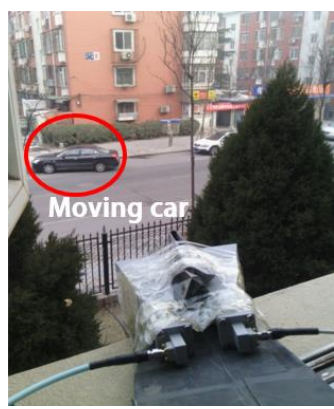

Figure 8. Target scenario: a moving vehicle.

Then ISAR echo data is acquired using the parameters listed in Table 1. The imaging process is performed in virtue of a motion compensation algorithm, by which each return range line is first aligned, then phase compensation based on the phase gradient autofocus (PGA) method is performed on each return. The imaging result of a moving vehicle on the scene is presented in Fig.9, the vehicle target was well focused and the outline is very clear, this indirectly demonstrates the good channel performance and phase consistency of the test platform. 


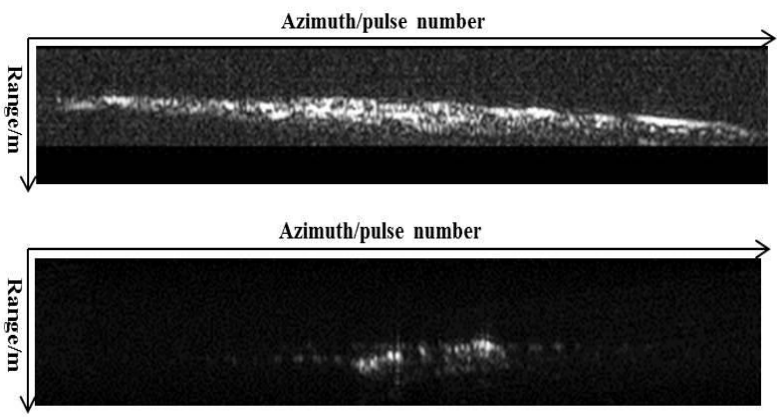

Figure 1. Envelope alignment and ISAR imaging result for a non-cooperative moving vehicle

\section{Conclusion}

A radar test platform is introduced. The platform is built upon commercial off-the-shelf test \& measurement equipment and custom hardware. It can test the performance of radar hardware modules by providing injection signals or simulate a radar transceiver. A control software is also provided to control the platform adaptively. At last, real experiment result is given to demonstrate the good performance of this platform.

\section{References}

1. Barton, D.K., Radar System Analysis (Dedham, MA: Bartech House, 1979)

2. Agilent Technologies, Agilent N6030A Series Arbitrary Waveform Generators User's Guide. (2006)

3. Agilent RF Signal Generator IVI-COM Driver Reference, Agilent Technologies, (2006)

4. Su F. L., Cao Z. D.. Improvements on the range alignment of the motion compensation for ISAR imaging, IEEE International Radar Conference. New York: 707-710 (1996) 